\title{
Dual-Light Photodynamic Therapy Effectively Eliminates Streptococcus Oralis Biofilms
}

\author{
Jessica Hentilä ${ }^{1}$, Noora Laakamaa ${ }^{1}$, Timo Sorsa ${ }^{1,2}$, Jukka Meurman ${ }^{1}$, Hanna Välimaa ${ }^{1,3}$, Sakari Nikinmaa ${ }^{4}$, Esko Kankuri ${ }^{5}$, \\ Tuomas Tauriainen ${ }^{6}$, Tommi Pätilä ${ }^{7}$ \\ ${ }^{1}$ Department of Oral and Maxillofacial Surgery, University of Helsinki and Helsinki University Hospital, Finland; \\ ${ }^{2}$ Department of Oral Medicine, Karolinska Institute, Huddinge, Sweden; ${ }^{3}$ Department of Virology, University of Helsinki, \\ Finland; ${ }^{4}$ Department of Neuroscience and Biomedical Engineering, Aalto University, Finland; ${ }^{5}$ Department of \\ Pharmacology, University of Helsinki, Finland; ${ }^{6}$ Heart and Lung Center, Meilahti Hospital, Helsinki, Finland; ${ }^{7}$ Department \\ of Congenital Heart Surgery and Organ Transplantation, New Children's Hospital, University of Helsinki, Finland
}

Corresponding author: Tommi Pätilä, MD, PhD, Otakaari 5 I 436, 02150 Espoo, Aalto University, Finland; TEL: +358 50427 2291; Fax: +358 94717 4479; email: tommi.patila@hus.fi

Received, June 6, 2021; Revised, September 10, 2021; Accepted, September 15, 2021; Published, September 22, 2021

ABSTRACT -- Purpose: During cancer treatment, oral mucositis due to radiotherapy or chemotherapy often leads to disruption of the oral mucosa, enabling microbes to invade bloodstream. Viridans streptococcal species are part of the healthy oral microbiota but can be frequently isolated from the blood of neutropenic patients. We have previously shown the antibacterial efficacy of dual-light, the combination of antibacterial blue light (aBL) and indocyanine green photodynamic therapy (aPDT). Methods: Here, we investigated the dual-light antibacterial action against four-day Streptococcus oralis biofilm. In addition, while keeping the total radiant exposure constant at $100 \mathrm{~J} / \mathrm{cm}^{2}$, we investigated the effect of changing the different relative light energies of aBL and aPDT to the antibacterial potential. Results: The dual-light had a significant antibacterial effect in all the tested combinations. Conclusion: Dual-light can be used as an effective disinfectant against $S$. oralis biofilm.

\section{INTRODUCTION}

Streptococcus oralis is a commensal species of viridans group streptococci (VGS) and part of the oral microbiota (1). S. oralis is an opportunistic pathogen, causing disease when the host defense mechanisms are conceded, and the infection routes become readily available. Typically, a disruption of oral mucosa during chemotherapy or radiotherapy leads to an enhanced risk of bacteremia. Thus, mucosal disruption, or oral mucositis, is not only a debilitating complication of cancer treatment but can severely hinder a patient's health and treatment outcome. Several reports indicate the increasing importance of VGS as the cause of bacteremia in immunocompromised patients (2). Increasing attention to different infection manifestations and antimicrobial resistance profiles within different involved VGS species, such as $S$. oralis and $S$. mutans, urges the need for examining the subspecies separately (3). Dual-light antibacterial therapy (aBL $405 \mathrm{~nm}$ and aPDT 810nm) of tissues that are reachable by the light, e.g., those in the mouth, is an emerging new treatment method, and its potential is now under research.
Dual-light antibacterial photodynamic therapy works on the basis of combination treatment, which has shown an efficient ability to eliminate $S$. mutans (4). The wavelengths used in the dual-light are 405 $\mathrm{nm}$ aBL and $810 \mathrm{~nm}$ near-infrared (NIR) light. The NIR light is used in combination with indocyanine green to produce the antibacterial action. Still, the same NIR is also absorbed by mitochondrial enzyme cytochrome-c-oxidase in eucaryotic cells. This absorption triggers a series of downstream effects, including improved ATP production. This action, called photobiomodulation exerts great potential in treating oral mucositis and has been widely recognized in clinical practice (5).

The dual-light can be used in the prevention and treatment of oral mucositis. The NIR light used in dual-light has shown an excellent clinical response to oral mucositis (6). We have previously shown the antibacterial effect of dual-light treatment against another VGS species, namely $S$. mutans, using different light energy combinations (4). Biological differences between the species of the VGS group prevent direct translation of the results between them, however. $S$. oralis can withstand high concentrations of hydrogen peroxide, which is not 
only a key antibacterial mechanism of aPDT action, but also the supposed mechanism of vulnerablility of streptococci to aPDT $(3,7)$. Consequently, in this study, we tested the efficacy of dual-light against $S$. oralis. In addition to the photobiomodulation effect, the antibacterial action of the dual-light might have an additional beneficial impact on the treatment outcomes of oral mucositis.

\section{MATERIALS AND METHODS}

Figure 1 describes a brief version of the study protocol. The incubation of $S$. oralis strain (ATCC 35037) was performed using a (NuAire DH autoflow 5500, NuAire Inc, US) equipment. The subsequent bacterial suspension was obtained by diluting it to a 2.9 McFarland concentration (Den 1 McFarland Densitometer, Biosan, Riga Latvia) using $0.9 \%$ saline solution. Three biofilm cultures were prepared for the control group and six biofilms for the experiment groups. Each biofilm was cultured for four days. A dual-light total radiant exposure of 100 $\mathrm{J}$ was applied for each biofilm with a specially prepared LED light applicator (Koite Health Oy, Espoo, Finland). Figure 1 describes the overview of dual-light antibacterial treatment mechanism of action. After the treatment, the biofilms underwent plating and further incubation for colony-forming unit (CFU) counting. The exact rates of irradiances between indocyanine green antibacterial photodynamic therapy (aPDT) $135 \mathrm{~mW} / \mathrm{cm}^{2}$ and antibacterial blue light (aBL) $42 \mathrm{~mW} / \mathrm{cm}^{2}$ are presented in Table 1. A detailed version of the study protocol can be found online (8), and the protocol, in brief, is shown in Figure 1.

- S. oralis bacteria were grown in BHI broth for 2 days.
- the bacterial suspension was diluted with $0.9 \% \mathrm{NaCl}$ to an optical density of 0.46 to
standardize the bacterial concentration in the suspension.
- $100 \mu \mathrm{g}$ of bacterial suspension was pipetted to each well used in a well plate. Six
repeats were used for each experiment.
- The bacteria were maturated for 4 days to form a solid biofilm.
- Standardized application of ICG photosensitizer was performed in each well, and
light application according to protocol was performed.
- After the light exposure, the entire biofilm from each well was collected, centrifuged,
and re-diluted for bacterial counting.
- Several serial dilution series were performed from each treated biofilm (from1:1 to
$1: 100000)$. Each dilution series were evenly spread to agar plates.
- Agar plates were left to incubate for $48 \mathrm{~h}$ for growth of the surviving bacteria.
- The colony forming units were counted. Typically, the plates showing bacterial count
between 30 to 800 is the most reliable dilution, from where the viable bacterial
number can be assessed using the inversed dilution ratio to multiply the actual
number of bacteria in the treated suspension.

Figure 1. Brief description of the study protocol.

\section{RESULTS}

After exposing the biofilms to the $100 \mathrm{~J} / \mathrm{cm}^{2}$ duallight photodynamic therapy, there were no detectable surviving $S$. oralis colonies. Three-to-one ratio of aPDT vs. aBL (aPDT $135 \mathrm{~mW} / \mathrm{cm}^{2}+\mathrm{aBL} 42$ $\mathrm{mW} / \mathrm{cm}^{2}$ ) successfully eradicated all $S$. oralis colonies, with a median CFU of 0 (range 0-0). Identical results were obtained after both one-to-one and one-to-three ratios of dual-light treatment, aPDT $79 \mathrm{~mW} / \mathrm{cm}^{2}+\mathrm{aBL} 73 \mathrm{~mW} / \mathrm{cm}^{2}$ and aPDT 38 $\mathrm{mW} / \mathrm{cm}^{2}+\mathrm{aBL} 130 \mathrm{~mW} / \mathrm{cm} 2$, respectively. The median number of CFUs in the control biofilms was $2.0 \times 10^{8}$ with a range of $1.9 \times 10^{8}-4.2 \times 10^{8}$. The results are presented in Table 1. Figure 2 shows the mechanisms of action regarding the dual-light treatment.

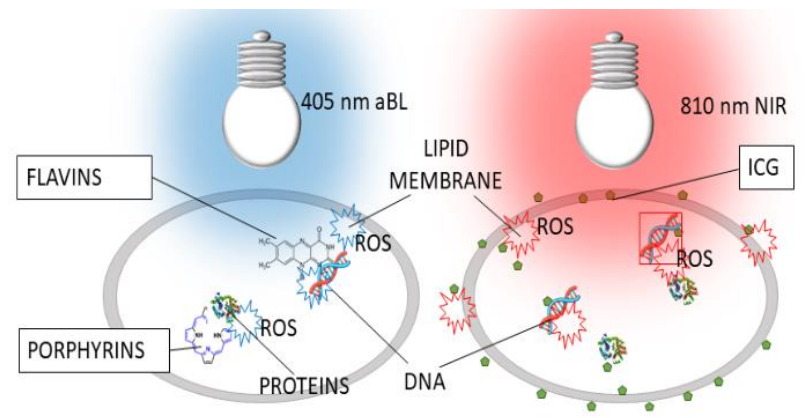

Figure 2. Dual-light antibacterial photodynamic therapy has both intrinsic and extrinsic action on S. oralis. The 405 $\mathrm{nm}$ light is absorbed by chromophores inside the bacteria, mostly by porphyrins and flavins. The absorbed energy can be transferred to nearby oxygen, producing reactive oxygen species (ROS). Indocyanine green (ICG) is used as an external photosensitizer in the method, causes external antibacterial action.

\section{DISCUSSION}

This study reveals the straight-forwarded effectiveness of dual-light against $S$. oralis, a commensal oral bacterium. In fact, we found no $S$. oralis colonies in the dual-light treated biofilms, which showed a disinfection level antibacterial effect compared to the control biofilms. The dosing of 100 $\mathrm{J} / \mathrm{cm}^{2}$ was effective at all combinations of $\mathrm{aBL}$ and aPDT. The biofilm had grown four days before the treatment, ensuring an appropriate and relevant maturation level. CFU method was used for counting the surviving bacteria for reliable and repeatable data.

We have previously tested different light energy combinations of dual-light against biofilms. 
Longer maturated biofilms are more susceptible to dual-light when the relative portion of aBL increases (4). The ability of aBL to infiltrate tissues is limited, while the NIR light can readily penetrate deeper into the mucosal tissue. Reducing the total light energy of NIR light decreases the possibility of overdosing, which can eventually impede the photobiomodulation effect (9). Thus, the antibacterial action against $S$. oralis in different duallight combinations enables low NIR dosing to mucosal tissue while keeping the antibacterial impact effectual.

Table 1. The results of three dose ratios of dual-light antibacterial therapy against Streptococcus oralis at a total energy density of $100 \mathrm{~J} / \mathrm{cm}^{2}$, measured as colony forming unit counts.

\begin{tabular}{|c|c|c|c|c|}
\hline & $\begin{array}{l}3: 1 \\
\left(\text { aPDT } 135 \mathrm{~mW} / \mathrm{cm}^{2}+\mathrm{aBL}\right. \\
\left.42 \mathrm{~mW} / \mathrm{cm}^{2}\right)\end{array}$ & $\begin{array}{l}1: 1 \\
\left(\text { aPDT } 79 \mathrm{~mW} / \mathrm{cm}^{2}+\right. \\
\left.\text { aBL } 73 \mathrm{~mW} / \mathrm{cm}^{2}\right)\end{array}$ & $\begin{array}{l}1: 3 \\
\left(\text { aPDT } 38 \mathrm{~mW} / \mathrm{cm}^{2}+\mathrm{aBL}\right. \\
\left.130 \mathrm{~mW} / \mathrm{cm}^{2}\right)\end{array}$ & Control biofilm \\
\hline $\begin{array}{l}\text { Streptococcus oralis, four- } \\
\text { day biofilm* }\end{array}$ & $0[0-0]$ & $0[0-0]$ & $0[0-0]$ & $\begin{array}{l}2.0 \times 10^{8}\left[1.9 \times 10^{8}-\right. \\
\left.4.2 \times 10^{8}\right]\end{array}$ \\
\hline Four-day biofilm nr. 1 & 0 & 0 & 0 & $2.0 \times 10^{8}$ \\
\hline Four-day biofilm nr. 2 & 0 & 0 & 0 & $1.9 \times 10^{8}$ \\
\hline Four-day biofilm nr. 3 & 0 & 0 & 0 & $4.2 \times 10^{8}$ \\
\hline Four-day biofilm nr. 4 & 0 & 0 & 0 & N/A \\
\hline Four-day biofilm nr. 5 & 0 & 0 & 0 & N/A \\
\hline Four-day biofilm nr. 6 & 0 & 0 & 0 & N/A \\
\hline
\end{tabular}

The results are presented as absolute colony forming unit counts. aPDT: antibacterial photodynamic therapy; aBL: antibacterial blue light; 3:1: Three-to-one ratio of aPDT compared to aBL; 1:1: One-to-one ratio of aPDT compared to aBL; 1:3: One-to-three ratio of aPDT compared to aBL; *: The results are presented as the median and range (in brackets) of colony forming unit counts of the study; N/A: Not available.

S. oralis has low pathogenicity, and it has been widely accepted to be a part of a healthy microbial community. However, high-dose chemotherapy and radiotherapy often lead to suppressed immune defense and oral mucositis, which change the pathogenic potential of bacteria. Untreated oral mucositis predisposes to bacteremia and severe systemic infectious complications. The treatment of oral mucositis with light-based methods has been shown very effective when used as biomodulation (5). When the oral immune fitness is weakened, antibacterial action can be used as another benefit of the light-based treatment. Thus, dual-light can provide a means to disinfect the mucositis lesions during the treatment, exemplified here by its excellent efficacy against $S$. oralis, and thus decrease the risk of bacteremia and microbial burden in patients with mucositis. The incidence of oral mucositis is very high in patients treated for hematologic, or head and neck cancer. These patient groups would be in the frontline to benefit from the antibacterial action of the dual-light. But, of course, the antibacterial ability of dual-light can be beneficial in the treatment of any local bacterial infections. Our current published results show the effectiveness of the method against $S$. mutans and $S$. oralis from the VGS group. These bacteria are mostly located in mouth, being part of the normal flora. Especially $S$. oralis can cause significant distant infectious complications such as endocarditis, but also maternal sepsis and neonatal sepsis, and meningitis.

\section{CONCLUSION}

Dual-light can be used as an effective disinfectant against $S$. oralis biofilm.

CONFLICT OF INTEREST. SN and TP are shareholders and members of the board in a company Koite Health Oy. Koite Health has filed patents P21233F100 and P22769F100, which are related to antibacterial dual-light. The company Koite Health is developing a dual light antibacterial product for the prevention and treatment of oral infections. JM has options in Koite Health Oy. TS is the inventor of 1274,416-patent U.S. 5,652,223, 5,736,341, $5,864,632,6,143,476$ and US 2017/0023571A1 (issued June 6, 2019), WO 2018/060553 A1 (granted 31.5.2018), 10,488,415 B2, Japanese Patent 2016554676, and South-Korean patent 10-2016-7025378.

AUTHOR CONTRIBUTIONS. SN and TP performed the microbial cultures, sample gathering and microbial cultivations; JH, NL, TS, JM, HV, SN, EK, TT, TP contributed to study design, analyzing the data, writing, and reviewing the manuscript. Jessica Hentilä and Noora Laakamaa contributed equally. 
FUNDING STATEMENT. The study has received financial support from Instrumentarium Foundation (Silmu Grant 2020).

\section{REFERENCES}

1. Zhou J, Sun T, Kang W, Tang D, Feng Q. Pathogenic and antimicrobial resistance genes in Streptococcus oralis strains revealed by comparative genome analysis. Genomics. 2020;5:3783-3793.

Doi:

10.1016/j.ygeno.2020.04.014.

2. Tunkel A, Sepkowitz K. Infections Caused by Viridans Streptococci in Patients with Neutropenia. Clin Infec Dis. 2002;11:15241529. doi: $10.1086 / 340402$.

3. Doern CD, Burnham CA. It's not easy being green: the viridans group streptococci, with a focus on pediatric clinical manifestations. $J$ Clin Microbiol. 2010;11:3829-3835. doi: 10.1128/JCM.01563-10. Epub 2010 Sep 1.

4. Nikinmaa S, Alapulli H, Auvinen P, Vaara M, Rantala J, Kankuri E, Sorsa T, Meurman J, Pätilä T. Dual-light photodynamic therapy administered daily provides a sustained antibacterial effect on biofilm and prevents Streptococcus mutans adaptation. PloS One. 2020;5 doi: 10.1371/journal.pone.0232775.

5. National Institute for Health and Care Excellence (NICE) (2018) Low-level laser therapy for preventing or treating oral mucositis caused by radiotherapy or chemotherapy (Interventional procedures guidance 615). Available at: https://www.nice.org.uk/guidance/ipg615

[Accessed: 20th of May 2021].

6. Bensadoun R-J, Nair R. Low-level laser therapy in the prevention and treatment of cancer therapy-induced mucositis: 2012 State of the art based on the literature review and meta-analysis. Curr Opin Oncol. 2012;4:363370 doi: 10.1097/CCO.0b013e328352eaa3.

7. Okahashi N, Nakata M, Kuwata H, Kawabata S. Streptococcus oralis Induces Lysosomal Impairment of Macrophages via Bacterial Hydrogen Peroxide. Infect Immun. 2016;7:2042-2050. doi: 10.1128/IAI.0013416.

8. The protocol for bacterial culturing in the present study at Protocols.io: dx.doi.org/10.17504/protocols.io.bfbcjiiw, Accessed on $10^{\text {th }}$ of May, 2021
9. Hamblin MR. Mechanisms and applications of the anti-inflammatory effects of photobiomodulation. AIMS Biophys. 2017;4(3):337-361. doi: 